\title{
Advances in Active Constraints and Their Application to Minimally Invasive Surgery
}

\author{
Stephen J. Starkie and Brian L. Davies \\ Imperial College of Science, Technology and Medicine, London SW7 2BX, UK, \\ s.starkie@ic.ac.uk
}

\begin{abstract}
This paper discusses advances in Active Constraints technology, which is designed to complement the surgeon's skill and improve safety in minimally invasive procedures. These advances include the support for true 3-dimensional constraints, independence of mechanical architecture, distributed constraint auditing, real-time deformation tracking and distributed user interface.
\end{abstract}

\section{Introduction}

In minimally invasive neurosurgery the endoscope has to pass through a burrhole in the skull, causing tool motions to be reversed in an occluded operating field. Active Constraints are intended to improve the safety of such reduced dexterity procedures, by implementing a virtual boundary which encloses the safe regions of the environment. To implement the constraint, an active robotic manipulator holds the tools and is guided under force-feedback control by the surgeon[1].

An important step in improving safety has been to split the control and interface of the system; VxWorks (x86) is used to control the manipulator, and Windows 2000 Pro(x86, accelerated graphics hardware) hosts the distributed user interface. Integration between the controller and the graphical interface is achieved using a proprietary $\mathrm{C}++\mathrm{CORBA}$ implementation.

\section{Active Constraints Implementation and Results}

Previous implementations of Active Constraints [1,2] utilised the specific multiplanar design of knee prostheses to define the boundary as sets of line segments on planes (earlier research [2] used B-Spline curves to define the planar geometry - but collision detection still involved a slow linear search). This method is insufficient in the general case, as boundaries with arbitrary 3-dimensional topography are desired. Triangular meshes are the most usual 3D surface representation, yet this is inelegant for two reasons; it presents a destabilising discontinuous gradients to the underlying force control structures, and accurate search methods 3 . for collisions of triangular meshes are too slow for real-time control. If more than two bodies are defined, Bounding Box techniques can be used to cull well seperated bodies, regardless of the underlying representation. 
Similarly, volumetric representations suffer from problems of discontinuity and slow search methods, despite allowing the possibility of directly mapping voxel intensities to physical properties, and easy implementation of cutting and tearing of bodies [4].

Parametric surfaces can be specified to be continuous to any order by simply specifying the order of the base polynomials, ensuring reasonable spacing of knots and providing enough control points. In this research, cubic B-Splines are used, as they include the property of local support (provided by the underlying B-Spline polynomials and which is lacking from Bezier surfaces). A B-Spline is a specialization of the more general class of curves called NURBS - Non-Uniform Rational B-Splines [5]. By specifying the knot vectors appropriately (ie; knot vectors are uniform) and the order as 3 , they can be guaranteed continuous up to second order.

The boundary search has been implemented as a non-terminating Newton Raphson minimisation of the closest approach vector (CAV) between two surfaces. The method has been implemented on a Neuro-Endoscopic Manipulator, and results have shown that although the iteration is not terminated (in order to continuously track the CAV) it converges after six iterations.

Forces are fed back to the manipulator control by implementing standard mechanical models; the boundary is modelled as a very hard spring, behind a low stiffness membrane and low viscosity damper.

\section{Conclusion}

This paper has described advances made in Active Constraint technology including the generalisation to 3D using cubic B-Spline boundaries. An extremely fast accurate collision detection algorithm has been developed which can operate in real-time. The technology has been implemented successfully in the control of an active Neuro-Endoscopic Manipulator.

\section{References}

1. S J Harris, M Jakopec, R D Hibberd, J Cobb, and B L Davies. Interactive preoperative selection of cutting constraints, and interactive force controlled knee surgery by a surgical robot. 1st Int. Conf. Medical Image Computing and Computer Assisted Intervention, pages 996-1006, 1998.

2. S C Ho, R D Hibberd, J Cobb, and B L Davies. Force control for robotic surgery. Proc. 7th Int. Conf. Advanced Robotics, 1:21-31, 1995.

3. M Lin. Efficient Collision Detection for Animation and Robotics. $\mathrm{PhD}$ thesis, University of California at Berkeley, 1993.

4. Sarah F Frisken-Gibson. Using linked volumes to model object collisions, deformation, cutting, carving, and joining. IEEE Transactions on Visualisation and Computer Graphics, 5(4), 1999.

5. Les Piegl and Wayne Tiller. The NURBS Book. Monographs in Visual Communication. Springer-Verlag, Berlin, 2 edition, 1997. 